TITLE:

\title{
Analysis of molecular dynamics of moist wood components by applying the stretched-exponential function
}

\author{
$\operatorname{AUTHOR}(\mathrm{S})$ :
}

Nakao, Sayaka; Nakano, Takato

\section{CITATION:}

Nakao, Sayaka ... [et al]. Analysis of molecular dynamics of moist wood components by applying the stretched-exponential function. Journal of Materials Science 2011, 46(14): 4748-4755

\section{ISSUE DATE:}

2011-02

URL:

http://hdl.handle.net/2433/141956

\section{RIGHT:}

The final publication is available at www.springerlink.com; この論文は 出版社版でありません。引用の際には出版社版をご確認ご利用くださ $\omega_{\circ}$; This is not the published version. Please cite only the published version. 
Analysis of molecular dynamics of moist wood components by applying the stretched exponential function

\section{Sayaka Nakao • Takato Nakano*}

Sayaka Nakao

Lineo Solutions , Inc., 1589-1 Kitaono, Shiojiri-Shi, Nagano 399-0651, Japan

Takato Nakano (* corresponding author)

Laboratory of Biomaterials Design, Division of Forest and Biomaterials Science, Graduate School of Agriculture, Kyoto University, Kita-Shirakawa, Kyoto, 606-8502 Japan

.e-mail; tnakano@kais.kyoto-u.ac.jp.

Key words stretched exponential function, wood, relaxation, water adsorption, hydrogen bond

\section{Abstract}

On the basis of the molecular dynamics of wood components, the effects of adsorption water molecules on the change of relaxation behavior were examined for wood specimens with various moisture contents using the low of mixture and stretched-exponential function (so-called the Kohlrausch-Williams-Watts (KWW) function). The KWW function has two parameters: the characteristic relaxation time and a parameter related to the dispersion of the relaxation time. Both parameters and the relaxation modulus at time zero, $E(0)$, could be separated into three regions corresponding to moisture contents of $0-0.05,0.05-0.10$, and greater than 0.10 . Experimental results provided a consistent explanation for the relationships between the isotherm curve analysis of wood, the dielectric relaxation behavior of adsorption water molecules, and the mechanical relaxation behavior of wood. 


\section{Introduction}

The relaxation behavior of wood has already been studied and many characteristic properties have been reported. The relationship between the relaxation behavior and moisture content is of particular interest because the physical properties, especially the mechanical and dielectric properties, relate to adsorption water molecules.

Carrington [1] reported that mechanical properties of wood change at a moisture content of about $5 \%$. This has already been confirmed by others such as Kollmann and Krech [2], Kajita et al. [3], and James [4]. The presumed reason for this is that adsorption water molecules form hydrogen bonds at lower moisture content levels, and form clusters and act as a plasticizer at higher moisture content levels. Dielectric relaxation measurements confirmed that adsorption water changes its bonding form with increasing moisture content $[5,6,7]$. However, the effects of water on the relaxation properties of wood and the relationship between the relaxation dynamics of adsorption water and that of wood have not yet been elucidated satisfactorily, even though the relaxation mechanism of adsorption water is fairly clear.

Discussion of the detailed molecular dynamics of wood is difficult because the relaxation properties for wood can not be examined on the basis of a characteristic function. The relaxation behavior of a synthesized polymer with a simple structure can be analyzed by linear viscoelastic theory and can be effectively described using a characteristic function such as distribution function or relaxation spectra. However, the same information for wood over a wide time region cannot be obtained because of the absence of a valid time-temperature superposition principle for a multiple system $[8,9,10]$. In addition, the relaxation spectrum even at each temperature is very broad and is not characteristic. Thus, little attention has been paid to the effects of various factors on the relaxation properties as they relate to the characteristic function. 
On the other hand, the stretched-exponential function (the KWW function) has been used to analyze the relaxation behavior of various materials. Though it is an empirical equation, it has used mainly in the analysis of dielectric relaxation because the relaxation behavior is described by just two parameters. Discussion on the basis of the KWW function, however, is not valid, if the non-exponential KWW function is not equivalent to the equation of the linear viscoelastic theory where the relaxation process is described by integration of exponential functions. Lindsey and Patterson [11] clarified the equivalence of both equations in this regard. Examination on the basis of the KWW function is thus worthwhile for wood, for which information over a wide time region cannot be obtained and the relaxation spectra are very broad and not characteristic.

In this work, the effects of hydrogen bonds created by adsorption water molecules on the relaxation behavior of wood are discussed using the KWW function, and the results of wood from a mechanical behavior standpoint are compared with the results obtained by dielectric measurements of water so that the relationship between the relaxation dynamics of adsorption water and that of wood is elucidated

\section{Experimental}

The rectangular wood samples used in the experiments were Japanese oak (Quercus crispula Blume) with no defects.

They measured $3 \times 10 \times 100 \mathrm{~mm}$ in the longitudinal, tangential, and radial directions, respectively. They were oven-dried overnight at $60^{\circ} \mathrm{C}$ under vacuum. They were then conditioned to a fixed moisture content in a chamber at $23^{\circ} \mathrm{C}$ with various saturated solutions for three weeks after drying.

The stress relaxation measurements were carried out in cantilever bending at $23^{\circ} \mathrm{C}$. The span and 
deflection were 60 and $2 \mathrm{~mm}$, respectively. This deflection was about 30\% of the linear upper limit for the air-dried sample and was applied to the RT-plane Deflection direction is approximately parallel to two components of wood, amorphous matrix (hemicellulose, and lignin) and microfibril (crystalline and amorphous cellulose) under this deflection condition. Samples were wrapped in polyethylene film to maintain constant moisture content. It was confirmed that measurement results was not influenced by wrapping.

\section{Results and discussion}

\section{KWW function and the linear viscoelastic theory}

The KWW function has been used to describe the dielectric relaxation behavior for many materials because of the good fit it provides. Using the KWW function, the mechanical relaxation modulus is represented by

$$
E(t)=E(0) \exp \left[-\left(\frac{t}{\tau_{0}}\right)^{\beta}\right]
$$

where $E(0)$ is the modulus at $t=0, t$ is the measurement time, $B$ is the "stretching parameter" that describes the distribution of relaxation time, and $\tau_{0}$ is the characteristic relaxation time, which is the time required to reach $1 / e$ of $E(0)$. The relaxation modulus at the infinite time $E_{\infty}$ is omitted in equation(1), which is generally zero for amorphous polymer. On the other hands, the linear viscoelastic theory describes the relaxation modulus by the following equation based on the Boltzmann superposition principle and causality,

$$
E(t)=\int_{0}^{\infty} \Phi(\tau) \exp (-t / \tau) d \tau=\int_{-\infty}^{\infty} H(\ln \tau) \exp (-t / \tau) d \ln \tau
$$

where $\Phi(\tau)$ is the distribution function and $H(\ln \tau)$ is the relaxation spectrum. The relaxation characteristic is 
confirmed using $\Phi(\tau)$ or $H(\ln \tau)$, so the study of the material behavior requires that they be calculated. The long-time asymptote value $E_{\infty}$ is not considered in the above equations.

Multiple component materials, especially materials such as wood with crystalline regions, differ from synthetic amorphous polymer in that they have a non-characteristic spectrum that is generally broad. The effects of various factors on the relaxation behavior of wood have therefore received little attention with regard to the relaxation spectrum. However, the KWW function appears suitable for the study of materials such as wood because the relaxation characteristics are controlled by two parameters.

If Eq. (1) is not equivalent to Eq. (2), the KWW function remains simply an empirical equation, even if the ability of Eq. (1) to describe many experimental results has led to its general acceptance. Lindsey and Patterson [11] showed the theoretical equivalence of these equations in this regard. They also showed that the distribution function $\Phi(\tau)$ is represented by an inverse Laplace transformation of $\exp \left[-(t / \tau)^{\beta}\right]$, and that it is uniquely confirmed by the parameters of the KWW function as follows:

$$
\Phi(\tau)= \begin{cases}-\frac{\tau_{0}}{\pi \tau^{2}} \sum_{k=0}^{\infty} \frac{(-1)^{k}}{k !} \sin (\pi \cdot \beta \cdot k) \Gamma(\beta \cdot k)\left(\frac{\tau}{\tau_{0}}\right)^{\beta \cdot k+1} & (\beta \neq 1 / 2) \\ \frac{\exp \left[-(1 / 4) \tau / \tau_{0}\right]}{2\left(\pi \cdot \tau \cdot \tau_{0}\right)^{1 / 2}} & (\beta=1 / 2)\end{cases}
$$

Lindsey and Patterson discussed the relationship between $\Phi\left(\tau / \tau_{0}\right)$ and $\tau / \tau_{0}$, and then noted that Eq. (3) is a convergence function. Moreover they derived the average relaxation time $<\uparrow>$ which is described in the following equation using $\tau_{0}$ and $B$

$$
<\tau>=\frac{\tau_{0}}{\beta} \Gamma(1 / \beta)
$$

where $I(1 / \not)$ is the Gamma function. 
Shamblin et al. [12] showed that the relaxation spectrum could be described using various functions when both equations were equal. These reports support the idea that the relaxation modulus described by the KWW function is an alternate description to that from linear viscoelastic theory. This study examined the moisture dependence of $B, \tau$, and $<\tau$ obtained using the KWW function and compared the results from previously reported dielectric measurements.

\section{Formulation of stress relaxation behavior of wood}

The discussion of mechanical properties of wood should be considered the high-order structure [13]. In our discussion, the following equation is adopted as the elastic modulus of the whole wood [14], because it seems to be the most simple formulation.

$$
\begin{aligned}
& E_{W}(t)=\left(\frac{\rho}{\rho_{s}}\right)^{m} E(t), \\
\therefore \quad & \ln E_{W}(t)=m \cdot \ln \left(\frac{\rho_{W}}{\rho}\right)+\ln E(t),
\end{aligned}
$$

where $E_{W}(t)$ and $E(t)$ are the modulus of the whole wood and wood substance, respectively, $\rho_{W}$ and $\rho$ are the density of the whole wood and wood substance, respectively, $\mathrm{m}$ is the structural parameter related to the porous structure of wood . Equation (6) can easily be derived by the use of the rule of mixture, considering wood as a porous materials consisting of the substance and the void. The time-dependent properties of the whole wood are reduced to that of the wood substance, because the first term in right side, that is the term related to shape factor of wood, is constant. Therefore, we will hereinafter discuss the elastic modulus of the wood substance.

Confirmation of parameters of the KWW function is difficult as for wood, which consists of multi components such as cellulose, hemicellulose, and lignin, so that the approximate procedure must be applied. However, 
applying to wood sample as a composite with two components of amorphous region (hemicellulose, lignin and amorphous cellulose) and crystalline cellulose, the relaxation modulus under our deflection condition is represented by

$$
\ln [E(t)]=\theta \ln \left[E_{a 0} \exp \left[-\left(\frac{t}{\tau_{a}}\right)^{\beta_{a}}\right]+E_{a \infty}\right]+(1-\theta) \ln \left[E_{c 0} \exp \left[-\left(\frac{t}{\tau_{c}}\right)^{\beta_{c}}\right]+E_{c \infty}\right]
$$

where $E_{0}$ and $E_{\infty}$ are the relaxation modulus at $t=0$ and $\mathrm{t}=\infty$, respectively, subscript "a" and "c" are amorphous region and crystalline microfibril in wood, respectively, $\theta$ is volume fraction of matrix and $\theta=0.75$. According to the low of mixture, Eq. (7) is valid when two components of a system are parallel to deflection direction. That holds under our measurement condition.

Now we consider the time region $t<<\tau_{c}$ because the characteristic relaxation time $\tau_{c}$ should be much more than measurement time due to that along crystalline microfibril. Additionally, considering $E_{a}(t) \gg>E_{a \infty}$ for amorphous matrix and little relaxation at $t<<\tau_{c}$ for crystalline microfibril, Eq.(7) is approximately reduced to

$$
\ln [E(t)] \approx \theta \ln \left[E_{a 0}\right]-\theta\left(\frac{t}{\tau_{a}}\right)^{\beta_{a}}+(1-\theta) \ln \left[E_{c 0}+E_{c \infty}\right]
$$

Equation (8) is more reduced to

$$
\ln \left[-\frac{d \ln [E(t)]}{\ln [t]}\right]=\left(\ln [\theta]+\ln \left[\beta_{a}\right]-\beta_{a} \ln \left[\tau_{a}\right]\right)+\beta_{a} \ln [t]
$$

Relationship between $\ln [-d \ln [E(t)] / d \ln [t]]$ and $\ln [t]$ gives the parameters $\tau_{\mathrm{a}}$ and $\beta_{\mathrm{a}}$ for amorphous matrix using $d \ln \left[E_{W}(t)\right] / \ln [t]=d \ln [E(t)] / \ln [t]$ because of the constant shape factor. Accordingly, the parameters of amorphous region can be obtained at least, though those of crystalline microfibril cannot be determined in the measurement period. Elucidation of the latter relaxation process should require the other analytical procedure. We will hereinafter discuss the relaxation behavior of amorphous components.

Validity of the above discussion cannot directly be examined by fitting experimental and theoretical results 
because of the following reasons. Equation (9) does not confirm all parameters necessary to describe a relaxation curve but those just for amorphous region. In addition, constants in equation (7) such as $E_{a \infty}$ and $E_{c \infty}$ are also unknown. Because the above procedure allows determine the required parameters by differentiating to eliminate their constant. However, the linearity of $\ln \left[-d \ln \left[E_{W}(t)\right] / d \ln [t]\right]$ and $\ln [t]$ can be examined. Figure 1 shows relationships between $\ln \left[-d \ln \left[E_{W}(t)\right] / d \ln [t]\right]$ and $\ln [t]$ in shorter time region for samples with typical moisture contents: broken and solid lines are experimental and theoretical results, respectively. Their relationships were found to be linear for them as estimated by Eq.(9). Regression line was calculated in the region $\ln [t]<10$, because Eq.(9) is approximately obtained under $t<<\tau_{c}$. This upper limit was determined considering the correlation. This limit value appears to be supported from results of relaxation spectra shown in Fig. 2(c), since this point divides the spectrum into two characteristic regions.

It should be noted that confirmation of parameters of the KWW function by commercial application software depend upon the initial conditions and that the appropriate condition is hardly found for wood. Under arbitrary condition, there is even a case that multiple sets of much different parameters confirm the best fit curve. The above procedure confirms the unique set of parameters for each measurement of wood.

\section{Relaxation behavior of wood at various moisture content levels}

Figure 2(a)-2(c) shows typical relaxation curves and relaxation spectra for various moisture contents, which is well-known result for wood samples. The relaxation spectrum is calculated by Alfrey's approximation. The curves for all samples initially decreased rapidly, followed by a more steady decrease, as shown in Fig.2(a). The modulus at $t=$ $10 \mathrm{~s}, E(10)$, depended on the moisture content. However, moisture dependence of the spectrum was not clear as shown 
in Fig.2(c), especially in the short time range. The experimental results obtained in this work were similar to those obtained in previous research. Fitting parameters were obtained from the linear regression in the shorter time region for all samples using Eq. (9) as mentioned before., so that the KWW function parameters $b_{\mathrm{a}}$ and $\tau_{\mathrm{a}}$ was calculated from their parameters.

Figures 3(a)-3(c) show the dependence of these parameters and $E(10)$ on the moisture content They had extreme values at a moisture content of 0.05 ; that is, $E(10)$ and $\tau_{\mathrm{a}}$ were maximum and $B_{\mathrm{a}}$ was minimum at a moisture content of 0.05 . The moisture-content dependence of $E(0)$ is similar to results reported by Carrington [1], Kollmann and Krech [2], and Kajita et al. [3]. The parameter $\tau_{\mathrm{a}}$ is the characteristic relaxation time, which is the time required to reach $1 / e$ of $E(0)$, i.e., the flexibility parameter of molecular chains of wood components. The $b_{a}$ is the distribution of relaxation times; $B_{a}=1$ implies a narrow range of relaxation times, that is, the single relaxation time while $b_{a}<1$ indicates a broad distribution. In other words, for a sample with a wider distribution of relaxation times, $B_{a}$ is less than unity.

Figures $3(\mathrm{a})$ and $3(\mathrm{~b})$ show that $E(0)$ and $\tau_{\mathrm{a}}$ are maximum and $B_{a}$ is minimum at a moisture content of 0.05 . This implies that the dynamics of amorphous matrix molecules changes at a moisture content of 0.05 . The parameter $B_{a}$ is generally about 0.5 for stress relaxation and about 0.3 for creep for many synthesized amorphous polymers [15]. Compared to the value of creep for many synthetic polymers, $B_{a}$ in this study seems to be too low. This is probably due to the application of the KWW equation to the wide time region. In any case, this small value shows that amorphous matrix of wood samples has a broad distribution of relaxation times corresponding to the broad relaxation spectrum.

The characteristic relaxation time $\tau_{\mathrm{a}}$ provides information about molecular dynamics of wood components. However, the average relaxation time $<\tau>$ depends on both $\tau_{a}$ and $b_{a}$, as mentioned above. In order to analysis 
dynamics of wood components in detail, $b_{a}$ as a function of $\tau_{a}$ or $<\tau_{a}>$ is examined, as shown in Figs. 4(a) and 4(b), where three regions can be defined. The moisture content boundaries between these regions are indicated in the figures. As $\tau_{\mathrm{a}}$ or $<\tau_{a}>$ increased, $b_{a}$ decreased in the moisture content region of $0.01-0.05$, and then increased for a moisture content of $0.05-0.10$. The relationship between $b_{a}$ and $\tau_{a}$ or $\left\langle\tau_{a}\right\rangle$ was approximately linear for moisture contents in the range $0.01-0.10$. On the other hand, the relationships for moisture content greater than 0.10 differed from that for less than 0.10 , that is, $b_{a}$ slightly decreased as $\tau_{a}$ or $<\tau_{a}>$ decreased. The effects of $\tau_{a}$ on $b_{a}$ were similar to those of $<\tau_{2}>$.

Figures $5(\mathrm{a})$ and $5(\mathrm{~b})$ show $E(0)$ as a function of $\tau_{\mathrm{a}}$ or $\left\langle\tau_{\mathrm{a}}>\right.$. Both relationships also had three separate regions corresponding to the moisture content ranges $0.00-0.05,0.05-0.10$, and $0.10-0.25$, as in Figs. 4(a) and 4(b). The separation of the regions in Fig. 6 was clearer than in Fig. 5

\section{Moisture content dependence of KWW parameters and relaxation mechanism of moist wood}

The unique situation that exists at a moisture content of 0.05 , where the modulus of wood was the maximum, may be due to hydrogen bonds created by adsorption water molecules as have been pointed by many researchers [1, 2,3$]$. However, no hard evidence of this has been provided to explain the mechanism for wood components. In particular, the relationship between the dynamics of wood components and adsorption water molecules is unclear because of the complex interaction between high order wood structure and adsorption water molecules [16, 17].

A group of Norimoto et al. [5, 6, 7] examined hydrogen bond creation by adsorption water and its dielectric relaxation mechanism with respect to the dynamics of adsorption water molecules. They clarified the dependence of activation energy on moisture content for the reorientation of adsorption water molecules based on thermodynamic 
considerations. Figure 6(a) is an example of the reorientation at lower moisture content $[5,6,7]$, which is similar to that of a water molecule in ice. This relaxation process shows that an adsorption water molecule creates four hydrogen bonds in wood and rotates by breaking three bonds. In this case, the activation energy is estimated to be $18.8 \times 3=$ $56.4 \mathrm{~kJ} / \mathrm{mol}$ (13.5 kcal/mol). Their experimental value was close to this model's value. On the other hand, Fig. 6(b) is an example with five hydrogen bonds at higher moisture content. Norimoto and Yamada [6] and Zhao et al. [7] reported that several water molecules created a loose hydrogen bond between adsorption sites at higher moisture content levels to form a cluster. This loose structure of hydrogen bond probably affects the flexibility of wood components.

In adsorption theories such as the dual mode theory that describes isotherm curves of various wood species $[18,19]$, two types of adsorption water are presumed to exist: hydration water and dissolution water. The ratio of the latter to the former depends upon the vapor pressure, which corresponds to the cluster size of adsorption water. Hydration water in wood is dominant at moisture content levels less than about 0.05 , while dissolution water is dominant at levels greater than about 0.10 , where hydration water levels off and dissolution water alone increases with increasing in vapor pressure [20]. Based on the dielectric measurement results by a group of Norimoto et al and the above discussion of adsorption water, we expect the form of adsorption water molecules in wood to change with the increase in vapor pressure as described below.

An adsorption water molecule creates four hydrogen bonds as the moisture content increases, that is, it directly bonds to four adsorption sites of the wood components for moisture contents in the range $0.00-0.10$, as shown in Fig. 6(a), according to Norimoto [5, 6]. This is referred to as hydration water and increases tight bonding during the adsorption process. On the other hand, clusters created by multiple molecules bond to adsorption sites of wood components so that the loose bonding increases as the moisture content increases, as shown in Fig. 6(b). This bonding 
structure probably provides flexibility to the wood components. For moisture contents greater than 0.10 , hydration water levels off and most of the adsorption water are dissolution water so that wood components have flexibility because of loose bonding in its moisture range.

The molecular motion of wood components is discussed based on the above discussion of the dielectric relaxation of adsorption water molecules. It should be noted that the molecular motion is of amorphous region. Figures 4 and 5 show that $b_{a}$ decreases and $\tau_{a}$ and $<\tau_{a}>$ increase with increasing moisture contents in the range 0.00-0.05. This implies that the relaxation time becomes longer and spreads over a broader spectrum as the moisture content

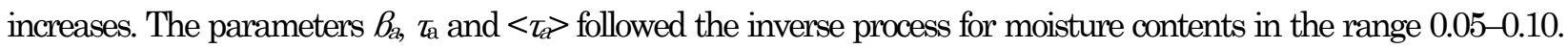
For moisture contents greater than $0.10, b_{a}$ decreased gradually, and $\tau_{a}$ and $<\tau_{a}>$ also decreased. The three regions in Figs. 4 and 5 clearly correspond to the results of the dielectric measurements reported by a group of Norimoto et al. Such a clear separation and the correspondence of the mechanical relaxation process shown in Figs. 4 and 5 have not been found in the analysis of the relaxation spectrum.

The average relaxation time $\left\langle\tau_{d}>\right.$ differs from the characteristic relaxation time $\tau_{\mathrm{a}}$ in that the former depends upon both $\tau_{a}$ and $b_{2}$, the latter reflects the relatively shorter relaxation process up to $1 / e$ of $E(0)$. The relationship between $<\tau_{\gtrsim}>$ and $\tau_{a}$ thus gives information during the change process of the relaxation time distribution. Figure 7 shows that the relationship between $<\tau_{d}>$ and $\tau_{a}$ consisted of two linear processes. The line slope at a moisture content of $0.00-0.10$ was larger than that at a moisture content greater than 0.10 . Both $\left\langle\tau_{a}>\right.$ and $\tau_{a}$ changed in the former moisture region, while $\tau_{a}$ alone changed in the latter region. This implies that the relatively shorter relaxation process up to 1/e of $E(0)$ changes in the moisture region greater than 0.10 . 


\section{Conclusion}

The effects of adsorption water molecules on the change of relaxation behavior were examined for wood specimens with various moisture contents and then discussed using stretched-exponential function, so-called the KWW function. This function has two parameters: the characteristic relaxation time and a parameter related to the dispersion of the relaxation time. Both parameters and the relaxation modulus at time zero, $E(0)$, could be separated into three regions corresponding to moisture contents of $0-0.05,0.05-0.10$, and greater than 0.10 . Experimental results provided a consistent explanation for the relationships between the isotherm curve analysis of wood, the dielectric relaxation behavior of adsorption water molecules, and the mechanical relaxation behavior of wood.

The above results differs from previous examinations of the relaxation behavior of wood in that it shows the analysis corresponding to both isotherm curves and dielectric measurements. Our results consistently relate adsorption behavior, dielectric relaxation, and mechanical relaxation. 


\section{References}

1. Carrington, H., (1922) The elastic constants of spruce as affected by moisture content. Aeron. J. 26: 462.

2. Kollmann, F., Krech, H. (1960) Dynamic measurement of damping capacity and elastic properties of wood. Holz als Roh- und Werkstoff 18:4-54.

3. Kajita, S., Yamada, T., Suzuki, M. (1961) Studies on rheological properties of wood. I. Mokuzai Gakkaishi, 7: 29-33.

4. James, W.L. (1961) Internal friction and speed in Douglas fir. F. P. J. 11(9) : 383-388.

5. Norimoto, M., Nakatsubo, F., Yamada, T. (1973) Dielectric properties of lignin. Zairyo (Journal of the Society of Materials Science, Japan) 22: 937-942.

6. Norimoto, M., Yamada, T. (1977) Dielectric behavior of water adsorbed on MWL. Mokuzai Gakkaishi (Journal of the Japan Wood Research Society) 23: 99-106.

7. Zhao, G., Norimoto, M., Yamada, T., Morooka, T. (1990) Dielectric relaxation of water adsorbed on wood, Mokuzai Gakkaishi (Journal of the Japan Wood Research Society) 36 : 257-263.

8. Fesco, D.G., Tschoegl, N.W. (1971) Time-temperature superposition in thermorheologically complex materials. J. Polym. Sci. 35: 51-69.

9. Kaplan, D., Tschoegl, N.W. (1974) Time-temperature superposition in two-phase polyblends. Polym. Eng. Sci. 14 : 43-49.

10. Nakano, T. (1995) Time-temperature superposition principle on relaxation behavior of wood as a multi-phase material. Holz als Roh- und Werkstoff, 53: 39-42.

11. Lindsey, C.P., Patterson, G.D. (1980) Detailed comparison of the Williams-Watts and Cole-Davidson function. J. Chem. Phys. 73: 3348-3357. 
12. Shamblin, S.L., Hancock, B.C., Dupuis, Y, Pinkal, M.J. (2000) Interpretation of relaxation time constants for amorphous pharmaceutical system. J. Pharmaceutical Sci. 89:417-427.

13. Salmén, L. and Burgert, I (2009) Cell wall features with regard to mechanical performance. A review, Holzforschung, 63, 121-129.

14. Ohgama, T. Yamada, T, (1974) Elastic modulus of porous materials. Mokuzai Gakkaishi 20:166-171.

15. Struik, L.C.E., "Physical Aging in Amorphous Polymers and Other Materials", Elsevier Scientific Publishing Company, Amsterdam, 1978.

16. Nakano, T. (2006) Analysis of the temperature dependence of water sorption for wood on the basis of dual mode theory. J. Wood Sci. 52: 490-495.

17. T. Nakano (2008) Analysis of cell wall swelling on the basis of a cylindrical model, Holtforschung, 62, 352-356 (2008).

18. Hailwood, A. J., S. Horrobin. (1946) Absorption of water by polymers: analysis in terms of a simple model. Trans. Faraday Soc. 42B: 84-102..

19. Michaels,A. S., Vieth W.R., Barrie,A. (1963.) Solution of gases in polyethylene Terephthalate. J.Appl. Phys. 34: $1-12$.

20. Tanioguchi, T., Nakato, K. (1978) Determination of water adsorption sites in wood by a hydrogen-deuterium exchange. Nature $272: 230-231$. 


\section{Captions}

Fig.1. Relationships between $\ln \left[-d \ln \left[E_{W}(t)\right] / d \ln [t]\right]$ and $\ln [t]$ for typical samples with three moisture content.

Broken and $\mathrm{s}$ lines are results calculated from experimental data and regression, respectively.

Fig.2. Typical stress relaxation curves ((a), linear scale; (b), logarithmic scale) and relaxation spectrum (c) for samples with various moisture contents.

Fig.3. Dependence of $\mathrm{E}(10), b_{a}$ and $\tau_{\mathrm{a}}$ on moisture content mc. Note: $b_{\mathrm{a}}$ is one of KWW function's parameters which represents the distribution shape of relaxation time, $\tau$ a is the characteristic relaxation time, $\langle\tau a$ is average relaxation time calculated from KWW function's parameters.

Fig.4. Relationships between $\beta$ a and $\tau_{\mathrm{a}}$ and $<\tau_{\mathrm{a}}>$. Note: $\beta$ a is one of KWW function's parameters which represents the distribution shape of relaxation time, $\tau_{\mathrm{a}}$ is the characteristic relaxation time, $\left\langle\tau_{\mathrm{a}}\right\rangle$ is average relaxation time calculated from KWW function's parameters.

Fig.5. Relationships between $\mathrm{E}(10)$ and $\tau_{\mathrm{a}}$ and $\left\langle\tau_{\mathrm{a}}>\right.$. Note: $\mathrm{E}(10)$ is a relaxation modulus at $\mathrm{t}=10, \tau_{\mathrm{a}}$ is the characteristic relaxation time, $<\tau_{a}>$ is average relaxation time calculated from KWW function's parameters.

Fig.6. Example scheme of the reorientation of adsorption water on wood material for an adsorption water molecule during hydrogen bond cutting (Norimoto and Yamada, 1973). 
Note: $\bar{\ominus}, \mathcal{\odot}^{\bullet}, \mathbf{\odot}$, and. $\bullet$. are wood substance, water molecule, hydroxyl group, and hydrogen molecule, respectively.

Fig.7. Relationship between $\tau_{\mathrm{a}}$ and $<\tau_{\mathrm{a}}>$. Note: $\tau_{\mathrm{a}}$ is one of KWW function's parameters which represents the characteristic relaxation time, $<\tau_{\mathrm{a}}>$ is average relaxation time calculated from KWW function's parameters. 


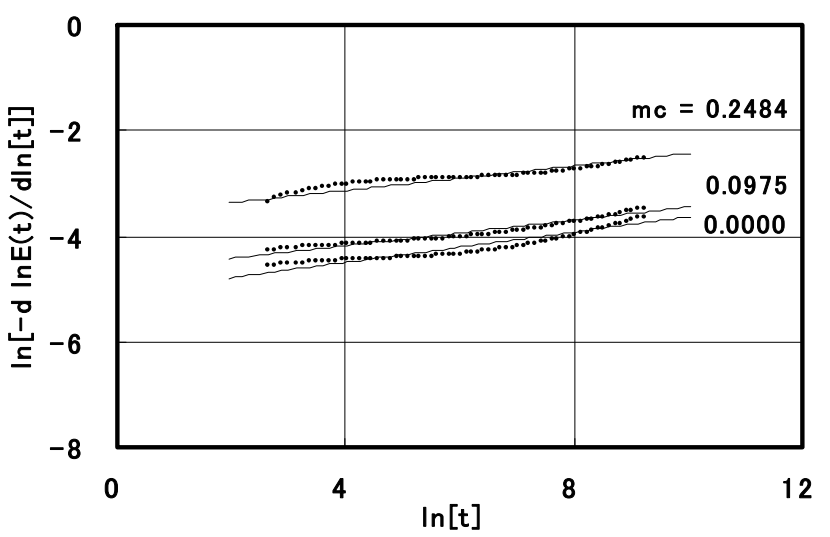

Fig.1. Relationships between $\ln \left[-d \ln \left[E_{W}(t)\right] / d \ln [t]\right]$ and $\ln [t]$ for typical samples with three moisture content. Broken and $\mathrm{s}$ lines are results calculated from experimental data and regression, respectively. 

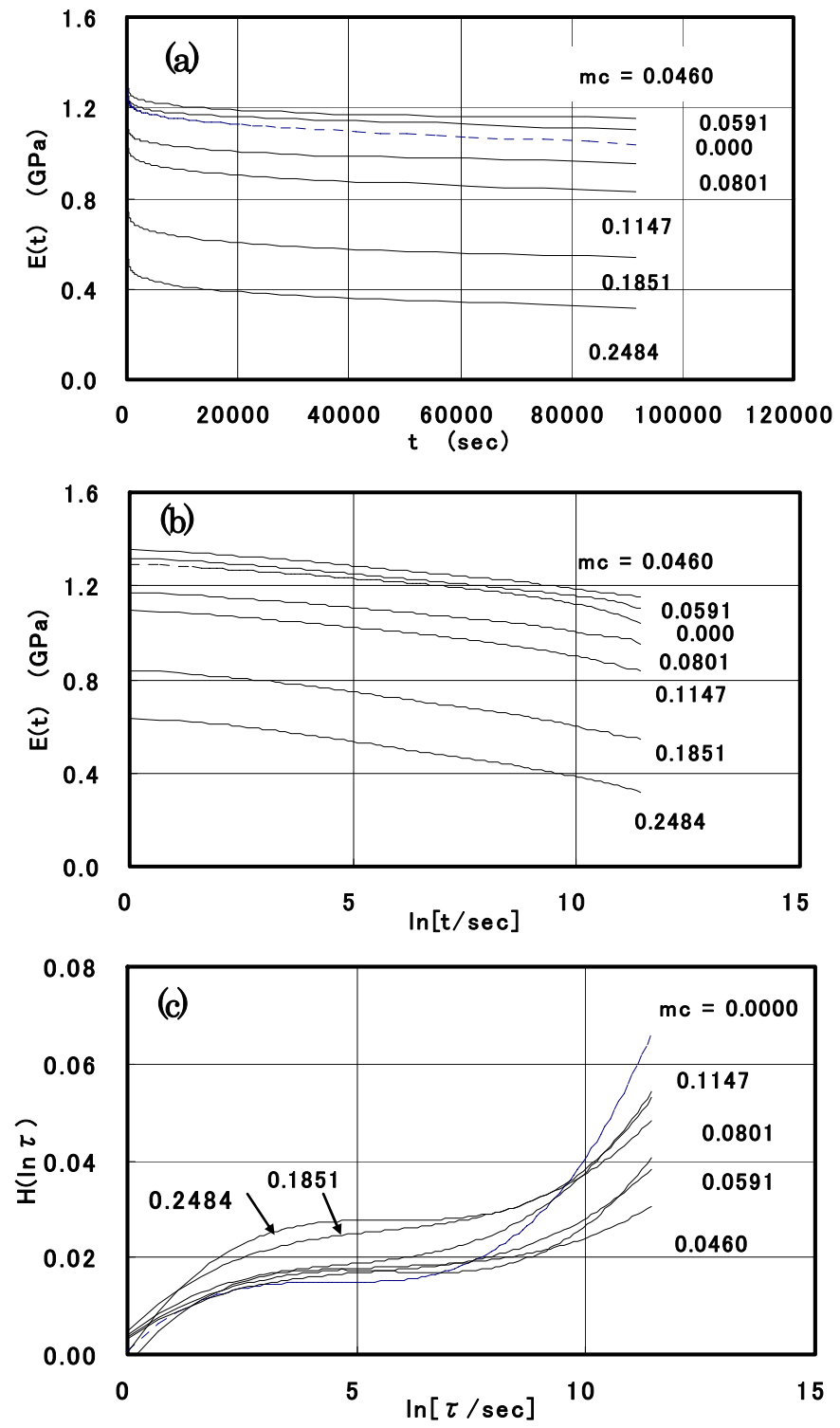

Fig.2. Typical stress relaxation curves ((a), linear scale; (b), logarithmic scale) and relaxation spectrum (c) for samples with various moisture contents. 

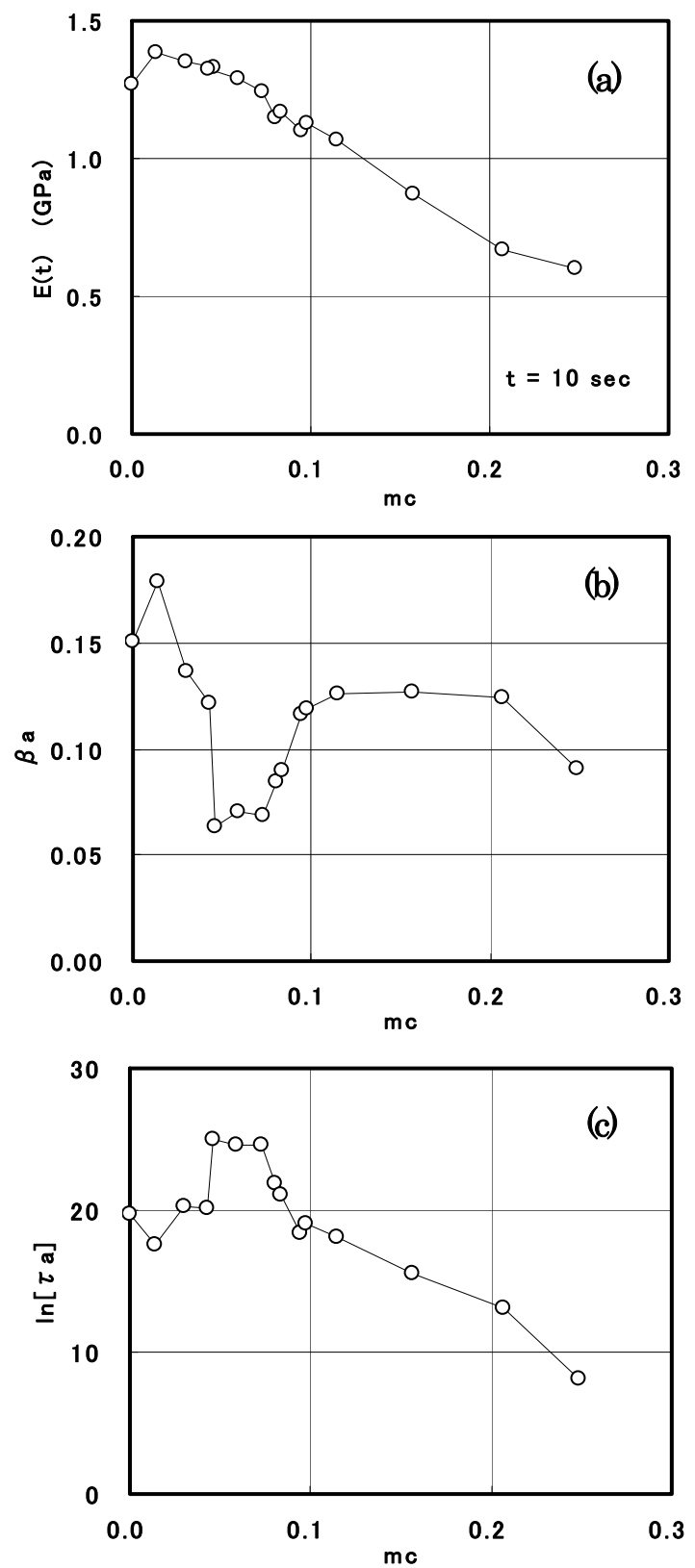

Fig.3. Dependence of $\mathrm{E}(10), \beta_{2}$ and $\tau$ a on moisture content mc. Note: $\beta$ a is one of KWW function's parameters which represents the distribution shape of relaxation time, $\tau_{\mathrm{a}}$ is the characteristic relaxation time, $\left\langle\tau_{\mathrm{a}}\right\rangle$ is average relaxation time calculated from KWW function's parameters. 

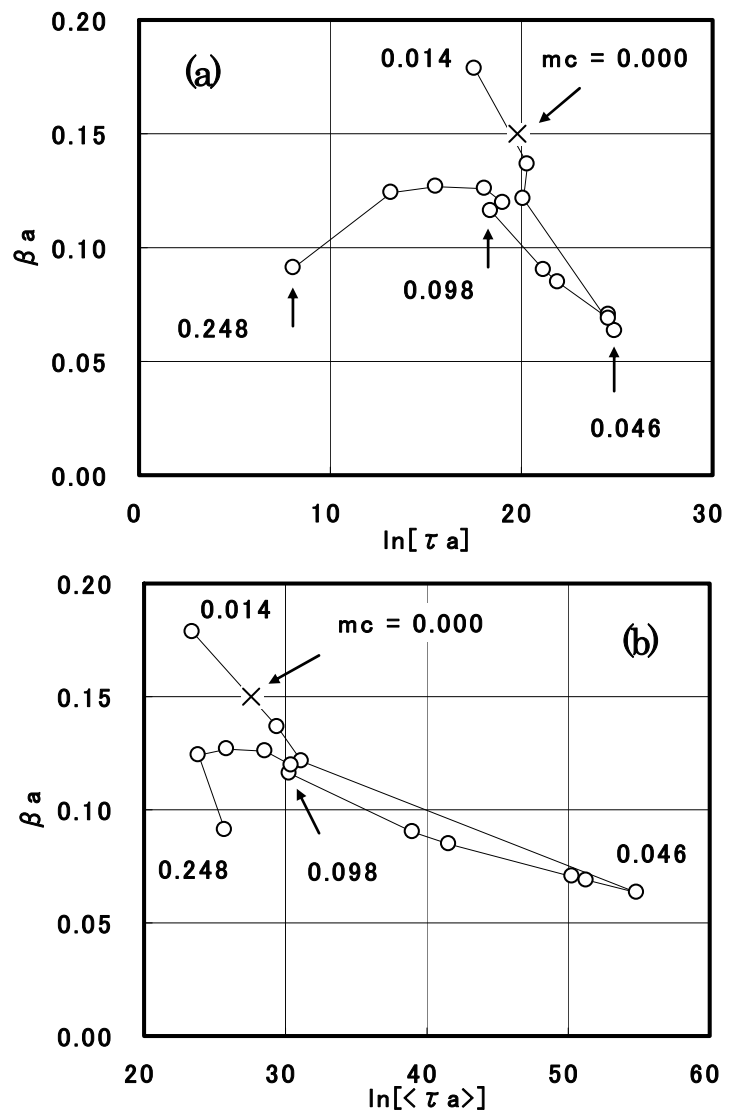

Fig.4. Relationships between $\beta$ a and $\tau_{\mathrm{a}}$ and $\left\langle\tau_{\mathrm{a}}>\right.$. Note: $\beta$ a is one of KWW function's parameters which represents the distribution shape of relaxation time, $\tau_{\mathrm{a}}$ is the characteristic relaxation time, $\left\langle\tau_{\mathrm{a}}>\right.$ is average relaxation time calculated from KWW function's parameters. 

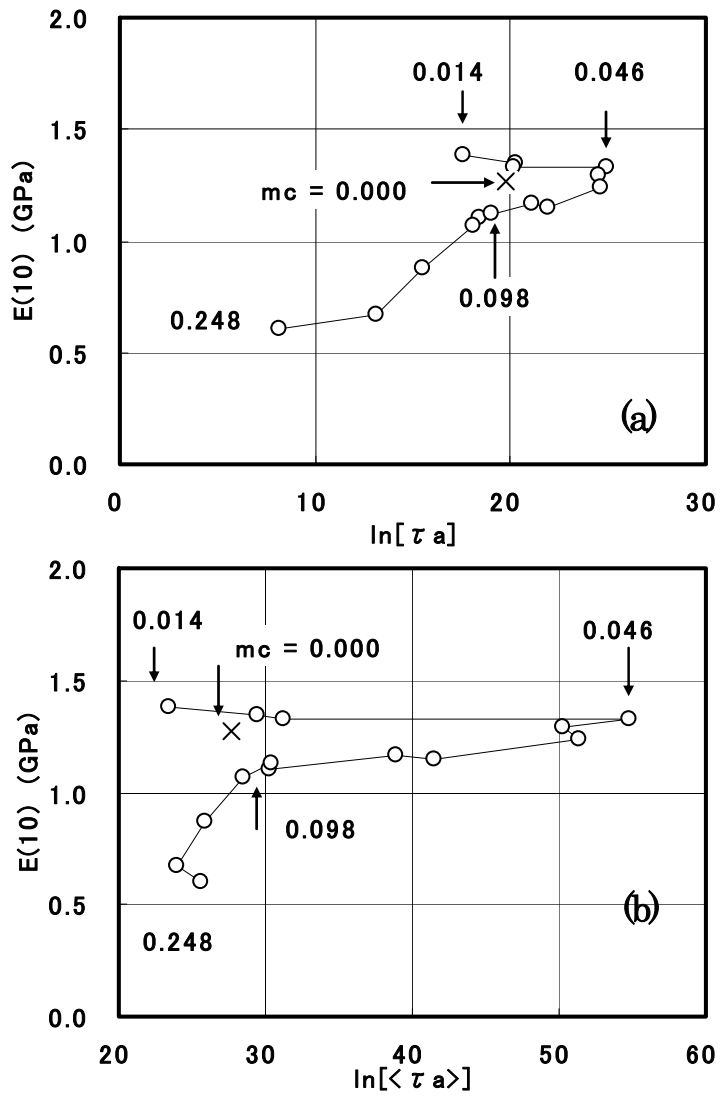

Fig.5. Relationships between $\mathrm{E}(10)$ and $\tau_{\mathrm{a}}$ and $\left\langle\tau_{\mathrm{a}}>\right.$. Note: $\mathrm{E}(10)$ is a relaxation modulus at $\mathrm{t}=10, \tau_{\mathrm{a}}$ is the characteristic relaxation time, $<\tau_{a}>$ is average relaxation time calculated from KWW function's parameters. 
(a)

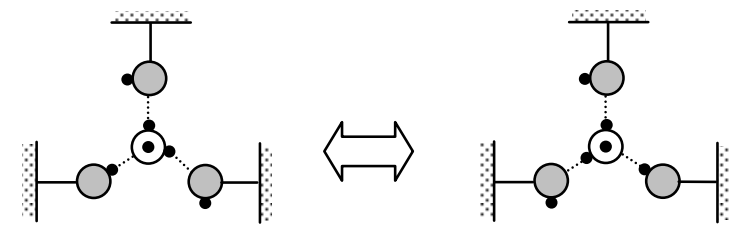

at lower moisture

(b)

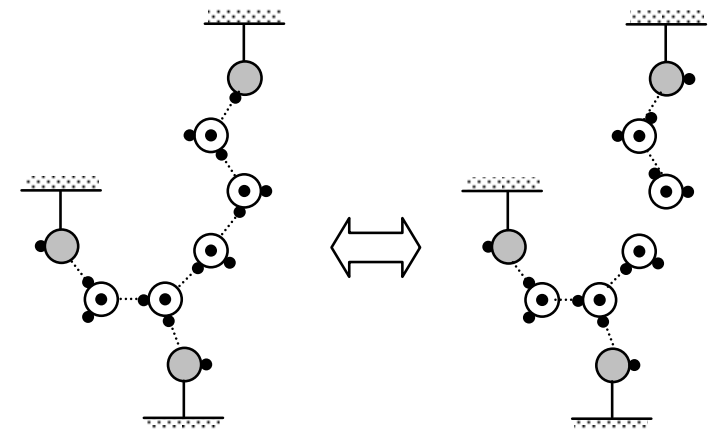

at higher moisture

Fig.6. Example scheme of the reorientation of adsorption water on wood substance for adsorption water molecule during cutting hydrogen bonds (Norimoto and Yamada, 1973).

Note: Symbols ॠ, ' $\bigcirc^{\bullet}, \quad \bigcirc$, and • are wood substance, water molecule, hydroxyl group, and hydrogen molecule, respectively. 


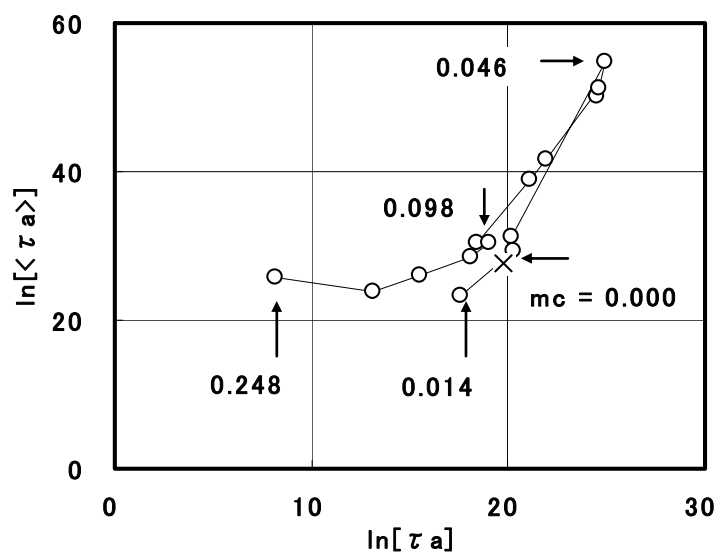

Fig.7. Relationship between $\tau_{\mathrm{a}}$ and $\left\langle\tau_{\mathrm{a}}>\right.$. Note: $\tau_{\mathrm{a}}$ is one of KWW function's parameters which represents the characteristic relaxation time, $<\tau_{\mathrm{a}}>$ is average relaxation time calculated from KWW function's parameters. 